\title{
Metabolic Surgery for Tackling Type II Diabetes Epidemic
}

\author{
*Siba Senapati \\ Consultant Bariatric Surgeon, USA
}

Received: September 20, 2017; Published: September 27, 2017

*Corresponding author: Siba Senapati, Salford Royal University Teaching Hospital, Honorary Senior Lecturer, University of Manchester, USA, Email: Siba.Senapati@srft.nhs.uk

\section{Introduction}

Type II Diabetes is a serious chronic metabolic dysfunction leading to increased morbidity, mortality and cost. Globally an estimated 422 million adults were living with diabetes in 2014, compared to 108 million in 1980 . The global prevalence of diabetes has nearly doubled since 1980 , rising from $4.7 \%$ to $8.5 \%$ in the adult population. This reflects an increase in associated risk factors such as being overweight or obese. Over the past decade diabetes has risen faster in low and middle-income countries than in highincome countries.

Diabetes caused 1.5 million deaths in 2012. Higher than optimal blood glucose caused an additional 2.2 million deaths, by increasing the risks of cardiovascular and other diseases. Diabetes Evolution of Metabolic Surgery is associated with significant complications which include heart attack, stroke, kidney failure, leg amputation, vision loss and nerve damage. Diabetes and its complications bring about substantial economic cost to people and health care system by its direct and indirect economic impact.

Although population based lifestyle interventions are essential to tackle current diabetes, people who develop diabetes should have access to all effective treatment options.

This is in part summary of Global Diabetes Surgery Summit II guidelines and endorsed by 50 National and International medical and metabolic surgery societies.

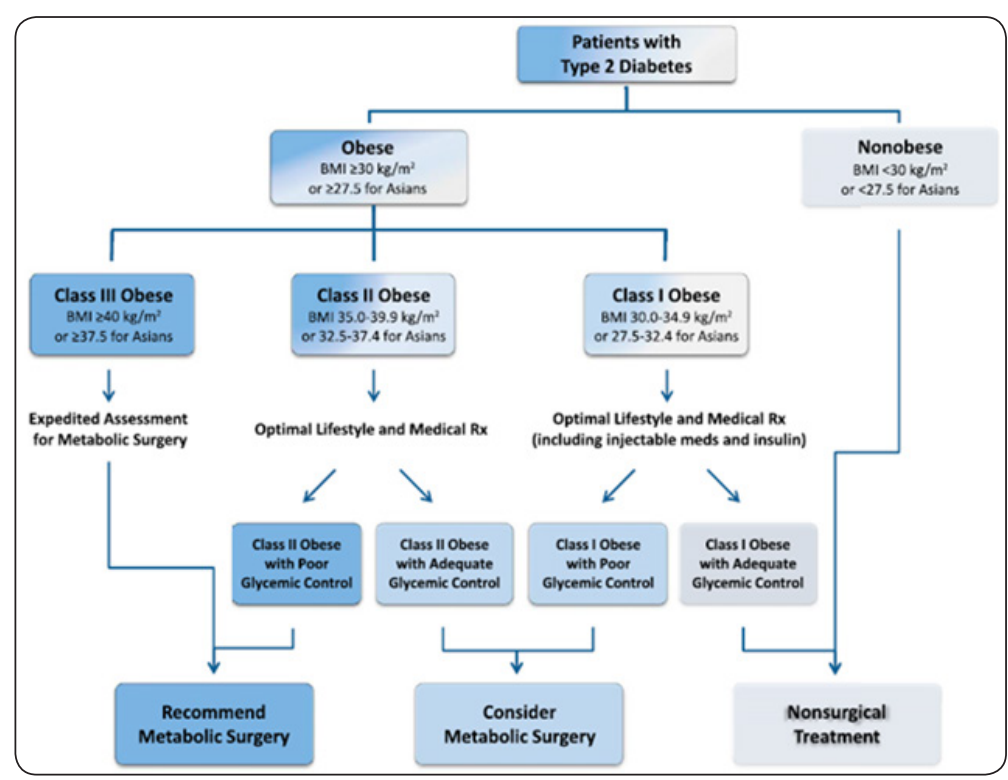

Figure 1: Algorithm for the treatment of T2D, as recommended by DSS-II voting delegates. The indications above are intended for patients who are appropriate candidates for elective surgery. Meds, Medications.

Metabolic surgery is defined as use of gastrointestinal operations with the intent to treat Type II diabetes and obesity. Observations that diabetes can be improved or resolved by surgical operations have been reported in the beginning of nineteenth century. Early reports in 1920's found that gastrointestinal operations for cancer or gastric ulcer caused dramatic improvement in diabetes. However after the advent of weight loss surgery in the 1950's evidence of diabetic remission were increasingly documented. 
Now experimental evidence and numerous randomised clinical trials performed over the last few decades, further reinforced that changes in gastrointestinal anatomy can directly influence glucose homeostasis leading to cure or remission of type II diabetes. The principle being metabolic surgery changes various mechanisms of GI physiology involved in glucose homeostasis. Improvement in diabetes occurs by increased insulin secretion, increased insulin sensitivity in the tissues, increase satiety and weight loss, alteration in bile acid pool and alteration in various gut hormones such glp-1, pyy, gip and ghrelin, etc (Fgures 1-4).

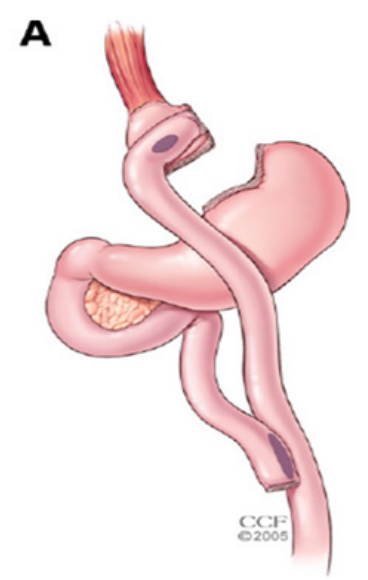

Figure 2 : Roux-en-Y Gatsric Bypass.

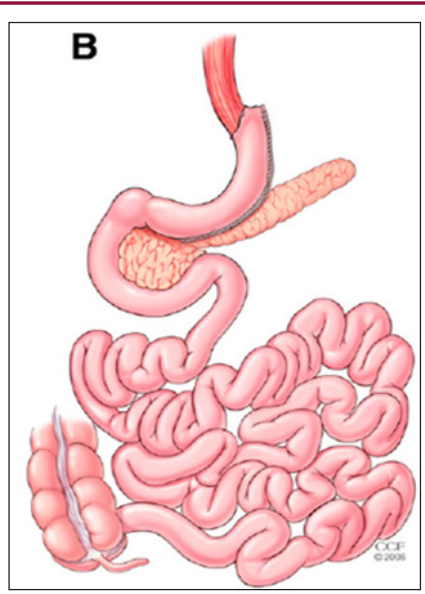

Figure 3 : Vertical Sleeve Gastrectomy.

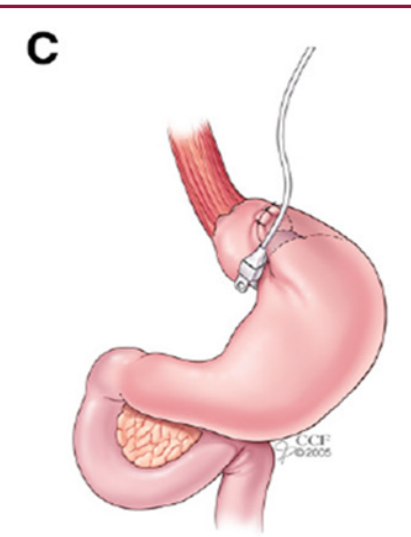

Figure 4 : Laproscopic adjustable Gastric Banding.

\section{Clinical Evidence}

11 randomised trials (Level-1 evidence) as well as large longterm case-controlled studies (level-2 evidence) comparing surgery in overweight/obese patients with type 2 diabetes show that metabolic surgery results in:

Greater improvement in glycaemic control

a. Reduction in medication use

b. Reduction in cardiovascular disease risk

c. Reduction in heart attacks, strokes, cancer and overall mortality
d. Greater weight loss
e. Better quality of life

\section{Diabetes Remission}

Evidence suggests that the chance of diabetes remission depends on duration of the disease ( higher if the duration is less than 5 years), is the pt only on medication or insulin or both, type of metabolic surgery carried out and c- peptide levels, which is an indirect measurement of pancreatic endocrine function status.

\section{Cost-effectiveness}

Multiple economic analyses have shown that surgical treatment for diabetes is cost-effective. Cost per quality adjusted life-year (QALY) is approximately $\$ 3200-6500$ well below $\$ 50000 /$ QALY.

With the introduction of minimal invasive or laparoscopic surgery over the last few decades, currently almost all of these procedures were performed with this approach, which leads to early recovery and shorter length stay. In fact many of the surgical procedures like Gastric Bypass, Sleeve gastrectomy and gastric banding are performed as ambulatory following the principles of enhanced recovery.

\section{Indications for Surgical treatment}

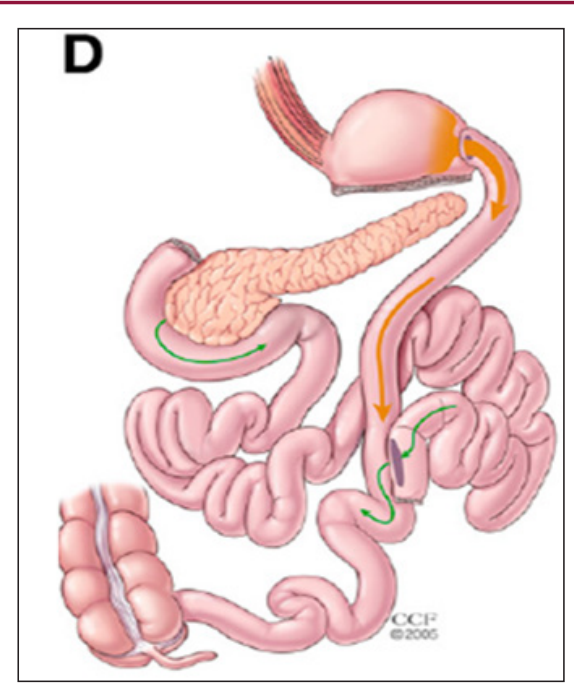

Figure 5 : Biliopancreatic Diversion.

There is now sufficient clinical and mechanistic evidence to support inclusion of metabolic surgery among anti-diabetes 
interventions for people with type II diabetes. Metabolic surgery should be a recommended option to treat Type II Diabetes in appropriate surgical candidates with class III obesity (BMI>_40KG/ $\mathrm{m} 2$ ) regardless of the level of glycaemic control or complexity of glucose lowering regimens as well as in patients with class II obesity (BMI $35-39.9 \mathrm{~kg} / \mathrm{m}^{2}$ ) with inadequately controlled hyperglycaemia despite lifestyle and optimal medical therapy.

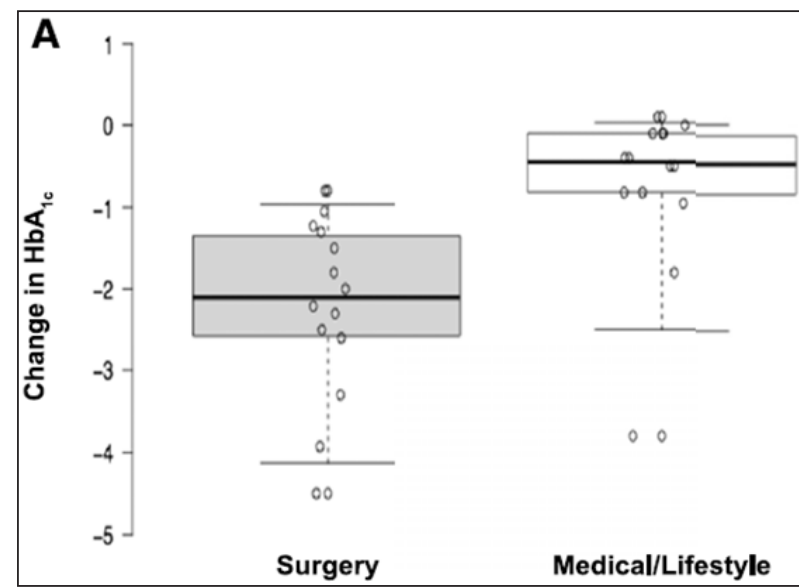

Figure 6 : Box plot comparing the average changes in HbA1c between surgery and medical/lifestyle treatments in the 11RCT's published.

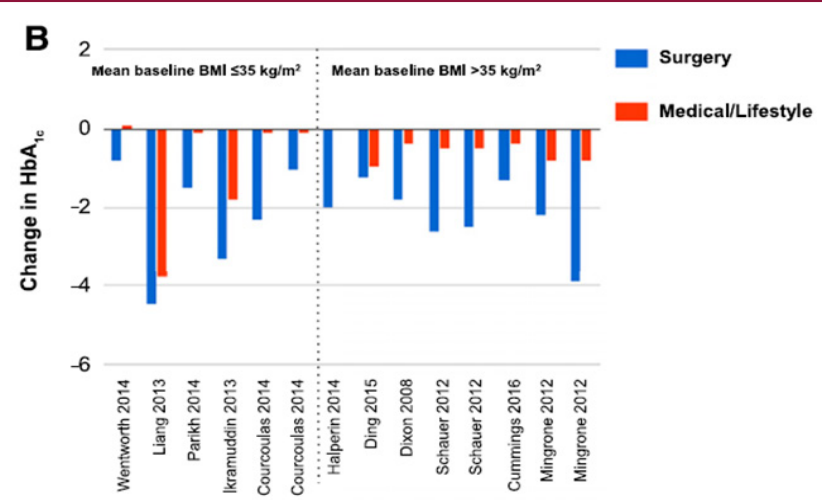

Figure 7 : Change from baseline $\mathrm{HbA1c}$ in each of the 11RCTs.

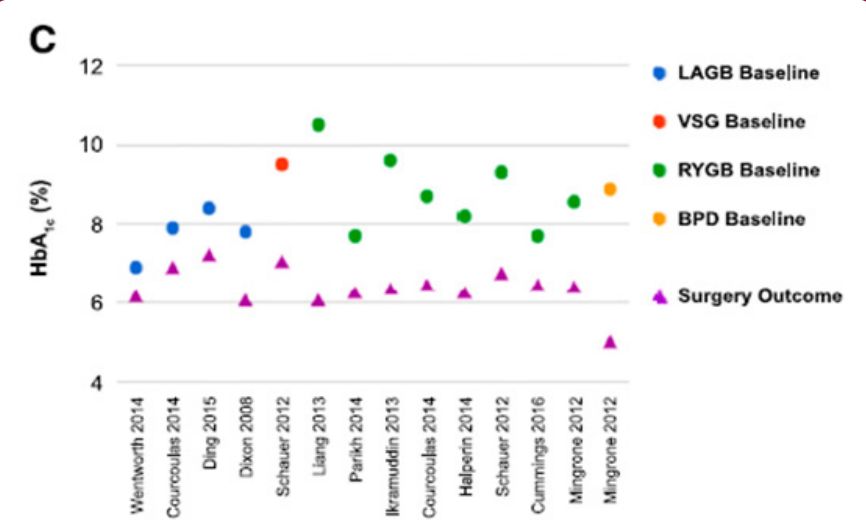

Figure 8 : Dot plot comparing baseline with final $\mathrm{HbA1c}$ levels following surgery in each of the 11RCTs.
Metabolic surgery should also be considered to be an option to treat Type II Diabetes in patients with class I obesity (30$34.9 \mathrm{~kg} / \mathrm{m}^{2}$ ) and inadequately controlled hyperglycaemia despite optimal medical treatment by either oral or injectable medications including insulin. For Asians the BMI thresholds should be reduced by 2.5 (Figures 5-8).

\section{Contraindication}

Contraindications to metabolic surgery include type I Diabetes unless surgery is indicated for obesity or other reasons, current drug or alcohol abuse, uncontrolled psychiatric illness, lack of comprehension of the risks and benefits and expected outcome. Commitment to nutritional supplements and long-term follow up is required for best outcome.

\section{Selecting the Surgical Procedure}

Out of the current four surgical procedures of gastric bypass, sleeve gastrectomy, gastric banding and bilio-pancreatic diversion, Gastric bypass appears to have a more favourable risk-benefit profile in most patients with Type II Diabetes.

\section{Safety and Complications}

Safety of bariatric and metabolic surgery has improved significantly over the last two decades with the exponential advances in minimal invasive surgery and enhanced recovery. Reported mortality risk is $0.1-0.5 \%$ similar to hysterectomy, cholecystectomy or hip replacement. Major perioperative complications are uncommon ranging from $2-6 \%$ and minor complications in up to $15 \%$. Long-term complications can occur with variable frequency depending on the metabolic surgical procedure.

\section{Follow-up}

Postoperative follow up should include surgical and nutritional evaluations at least every 6 months and more often if necessary during the first two post-operative years and at least annually thereafter. Even if patients experience diabetes remission, monitoring of glycaemic control should be continued with the same frequency as recommended for patients with prediabetes because of the potential for relapse. Long-term monitoring of micronutrient status, nutritional supplementation and support must be provide to patients after surgery according to national and international societies guidelines. 


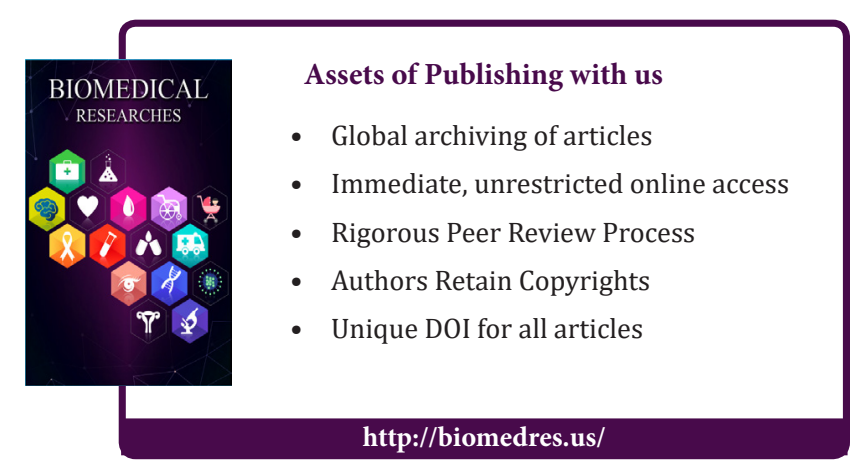

